\title{
China's new WHO flu monitoring center seeks to reverse criticism
}

BEIJING - China has not always been a world leader when it comes to infectious disease surveillance. Severe acute respiratory syndrome caught the country by surprise in 2003 , and, two years later, government officials went into denial after reports surfaced that H5N1 avian influenza had infected people and birds. But since those debacles, China has ramped up its screening efforts, building several infectious-disease institutes and more than 400 labs devoted to flu surveillance and testing, plus adding sentinel equipment to some 550 hospitals. So when H1N1 'swine flu' struck four years later, the world's most populous country was much better prepared.

"China has set up the world's largest influenza surveillance network," Yuelong Shu, director of the National Influenza Center, part of the Chinese Center for Disease Control and Prevention (CDC), told Nature Medicine. And now, China can also boast being the first country in the developing world to host a World Health Organization (WHO) Collaborating Center for Reference and Research on Influenza.

Joining other collaborating centers in Australia, Japan, the UK and the US, the Beijingbased National Influenza Center will serve as a regional hub for monitoring and responding to flu outbreaks. The Chinese center will also host research into new antiviral medicines and help provide pandemic preparedness training for medical personnel from across East Asia.

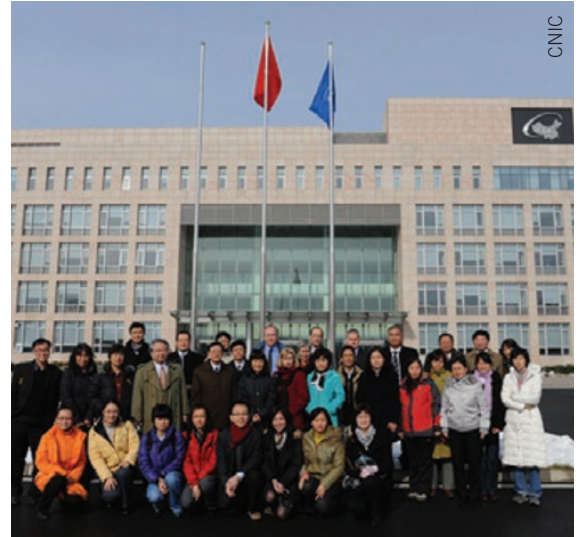

Flu-ent Chinese: The National Influenza Center.

“This designation reflects the country's exceptional contribution to the global fight against influenza," Young-soo Shin, the WHO's regional director for the western Pacific, said at a designation ceremony here on $14 \mathrm{March}$, the state-owned China Daily newspaper reported.

China's surveillance efforts weren't always so laudable. Just five years ago, the country's lack of transparency and preparedness in dealing with the avian flu crisis drew sharp criticisms from WHO officials. "But the situation has improved dramatically in recent years," says Qiyong Liu, an infectious disease expert at the Chinese CDC's National Institute for Communicable
Disease Control and Prevention. "Now China is quite open to the outside."

On a domestic level, Liu also thinks that the National Influenza Center's status as a WHO organ-complete with data sharing between the five global centers-will promote a spirit of greater cooperation among the Chinese research community. "The reference center will help Chinese institutions and pharmaceutical companies get influenza viral strains and other data more quickly and more comprehensively, facilitating their work to develop vaccines and therapies against particular viral strains," he says.

Even so, gaps still remain in China's monitoring. According to a recent report by leading officials at the Chinese CDC and its US counterpart, China still needs to invest more in pathogen-based surveillance systems such as the US CDC's PulseNet to contain outbreaks before they cause substantial illness and death (Health Aff. 30, 127-135, 2011). "The clinical hospitals should work together with our CDC system to isolate and identify new pathogenic strains that could cause new epidemics," says study coauthor Zijian Feng, director of the Office of Disease Control and Emergency Response at the Chinese CDC. "We are proposing epidemic and public health policy revisions to promote and support this development."

Hepeng Jia

\section{Qatar proposes national council to direct research efforts}

On 3 April, Qatar unveiled its first National Health Strategy (NHS), which covers the next five years and includes a plan to launch a new national governance body to better manage resources and projects across the various biomedical centers in the small Persian Gulf state. The newly proposed Qatar Medical Research Council (QMRC) will be based in Doha and will be responsible for coordinating research efforts between institutions and communicating the scientific outcomes to policymakers.

Currently, most of the scientific work taking place in Qatar is in basic biomedical research, and in 2006 the country committed to raising science funding to $2.8 \%$ of its gross domestic product. "Given the generous resources and the unwavering strive to excellence, it is worthwhile considering how to enhance the current elements involved in biomedical science and health research in Qatar," says Momtaz Wassef, a former director of Qatar's Department of Biomedical Research at the Supreme Council of Health who advised on the new NHS plan.

The fast growth in Qatar's investment in science has led to the establishment of several large-scale research centers, such as the healthcare facilities run by the Hamad Medical Corporation and a branch of the New York-based Weill Cornell Medical College. There is hardly any coordination among such sites, however, which the NHS suggests could be leading to duplication of efforts and wasted resources.

"We propose QMRC as a model to maximize the impact of investment in biomedical and health research," explains Wassef, who is now an $a d$ hoc advisor at the US National Institutes of Health (NIH).

He adds, though, that the new system should "stay respectful of individual institutions' ethos."
The current funding bodies "lacked strategic oversight and did not obviously connect to the needs of the Supreme Health Council," says David Kerr, professor of cancer medicine at University of Oxford in the UK and a past member of the executive committee for Qatar's 2011-2016 NHS.

"Diabetes is an obvious area of study that should be addressed as one of the priority programs instigated by the new council," he adds.

Kerr notes that the new QMRC is not designed to replace current funding schemes that focus on curiositydriven, basic research. "This would be complementary to existing funding streams and would not detract from any current investment in more basic science," he says.

Mohammed Yahia 